\title{
Biosynthesis and Identification of Metabolites of Maraviroc and Their Use in Experiments to Delineate the Relative Contributions of Cytochrome P4503A4 versus $3 \mathrm{A5}^{\text {政 }}$
}

\author{
Elaine Tseng, Gwendolyn D. Fate, Gregory S. Walker, Theunis C. Goosen, and R. Scott Obach \\ Pfizer Inc., Groton, Connecticut
}

Received December 4, 2017; accepted February 21, 2018

\begin{abstract}
Maraviroc (MVC) is a CCR5 coreceptor antagonist indicated in combination with other antiretroviral agents for the treatment of CCR5-tropic human immunodefinciency virus-1 infection. In this study, the metabolism of MVC was investigated in human liver microsomes to delineate the relative roles of CYP3A4 and CYP3A5. MVC is metabolized to five hydroxylated metabolites, all of which were biosynthesized and identified using mass and NMR spectroscopy. The sites of metabolism were the 2- and 3-positions of the 4,4-difluorocyclohexyl moiety and the methyl of the triazole moiety. Absolute configurations were ultimately ascertained by comparison to authentic standards. The
\end{abstract}

biosynthesized metabolites were used for quantitative in vitro experiments in liver microsomes using cyp3cide, a selective inactivator of CYP3A4. (1S,2S)-2-OH-MVC was the main metabolite representing approximately half of the total metabolism, and CYP3A5 contributed approximately $40 \%$ to that pathway in microsomes from CYP3A5*1/* 1 donors. The other four metabolites were almost exclusively metabolized by CYP3A4. (1S,2S)-2hydroxylation also correlated to T-5 $\mathrm{N}$-oxidation, a CYP3A5-specific activity. These data are consistent with clinical pharmacokinetic data wherein CYP3A5 extensive metabolizer subjects showed a modestly lower exposure to MVC.

\section{Introduction}

Maraviroc (MVC) is a human chemokine (C-C motif) receptor 5 (CCR5) antagonist indicated in combination with other antiretroviral agents for the treatment of CCR5-tropic human immunodefinciency virus (HIV)-1 infection (Dorr et.al., 2005). HIV-1 strains use CCR5 as a coreceptor during the transmission stage and in the early stages of HIV disease (Michael et al., 1997; Philpott, 2003). Blocking the CCR5 receptor prevents entry of HIV into host cells and is shown to reduce viral load to undetectable levels in both treatment-naive and treatmentexperienced populations and is sustained out to 5 years of follow up. MVC is rapidly absorbed and extensively metabolized, although unchanged MVC is the major circulating component in plasma and is the major excreted component after oral dosing. Previous in vitro experiments showed that MVC undergoes oxidative metabolism mediated by CYP3A4 (Hyland et al., 2008). Renal clearance contributes $23 \%$ of total clearance and the absolute bioavailability of $100 \mathrm{mg}$ oral MVC dose is 23\% (estimated at $33 \%$ for $300 \mathrm{mg}$ dose) (Abel et al., 2008, 2009; Vourvahis et al., 2013). A recent publication by Lu et al. (2014) described the effects of the CYP3A5 genotype on MVC plasma concentrations. Those results suggested that CYP3A5 may play a prominent role in the metabolism of MVC in subjects with functional CYP3A5 alleles. This was in line with data in human liver microsomes (HLMs) showing that the estimated CYP3A5 contribution to MVC metabolism in HLMs from wild-type CYP3A $5 * 1 / * 1$ donors [extensive

https://doi.org/10.1124/dmd.117.079855.

S This article has supplemental material available at dmd.aspetjournals.org. metabolizers (EMs)] was $32 \%$ compared with only $2 \%$ in HLMs from CYP3A5*3/*3 donors (poor metabolizers) (Tseng et al., 2014).

In this paper, we describe the biosynthesis and identification of four metabolites that are hydroxylated on the difluoro cyclohexyl moiety, out of eight possible regio- and stereoisomers, along with a fifth wherein hydroxylation is on the methyltriazole moiety. The contribution of CYP3A5 to their formation was quantitatively delineated through the use of pooled HLMs from CYP3A $5 * 1 / * 1$ genotype donors, the CYP3A4 selective inactivator cyp3cide (Walsky et al., 2012), the CYP3A5 selective reaction T-5 N-oxidation (Li et al., 2014), and recombinant heterologously expressed CYP3A4 and CYP3A5.

\section{Materials and Methods}

Materials. MVC, $\mathrm{d}_{5}$-MVC, T-5 [methyl 2-(4-aminophenyl)-1-oxo-7-(pyridin2-ylmethoxy)-4-(3,4,5-trimethoxyphenyl)-1,2-dihydroisoquinoline-3-carboxylate], T-5 $\mathrm{N}$-oxide, and cyp3cide were prepared at Pfizer (Groton, CT). OH-MVC and deuterated $\mathrm{OH}-\mathrm{MVC}$ isomers were biosynthesized as described subsequently; chemical synthesis of all eight 2- and 3-OH-MVC isomers are described in the Supplemental Material. Ketoconazole, terfenadine, and NADPH were obtained from Sigma-Aldrich (St. Louis, MO). A 50-donor pool of HLMs (HLM-102; equal females and males) was prepared under contract by BD Gentest (Woburn, MA). Single genotyped HLMs used in this study are similar to those from Walsky et al. (2012). CYP $3 A 5^{*} 1 / * 1$ : HH47, BD HH785, BD HH867, BD HH860, HH86, HH103, HH104, and HH107; CYP3A5*1/*3: HH1, HH2, HH8, HH9, HH80, HH89, HH90, HH91, HH92, HH108, HH117, HH48, and HH100; and CYP3A5*3/*3: BDHH189, HH25, HH116, HH118, HH74, HH75, and HH98 were obtained from BD Biosciences (San Jose, CA). The BD designation in front of some microsomal donor samples indicates the sample has been genotyped and immunoquantified for CYP3A4 and CYP3A5 by BD Biosciences; otherwise, the

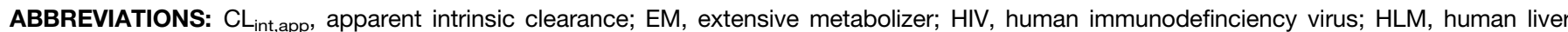
microsome; HPLC, high-performance liquid chromatography; HSQC, heteronuclear single quantum coherence; LC-MS/MS, liquid chromatographytandem mass spectrometry; MS, mass spectrometry; MVC, maraviroc; P450, cytochrome P450; UHPLC, ultra-high-performance liquid chromatography. 
donor characterizations were conducted by Pfizer. Pooled lots of HLM CYP3A $5 * 1 / * 1$ (from five donors; two females, three males) and HLM CYP3A5*3/*3 (from six donors; one female, five males) were prepared by pooling equal volumes of some of the aforementioned individual lots such that the resulting enzymatic activity (measured by $6 \beta-\mathrm{OH}$ testosterone formation) is similar between the two lots. Recombinant heterologously expressed CYP3A4 and CYP3A5 were prepared under contract by Panvera Corp. (Madison, WI). All other chemicals and reagents were from standard suppliers.

Metabolism of Maraviroc by Recombinant CYP3A4 and 3A5. MVC $(10 \mu \mathrm{M})$ was incubated with recombinant human CYP3A4 or CYP3A5 both at $100 \mathrm{pmol} / \mathrm{ml}$, in $100 \mathrm{mM}$ potassium phosphate buffer ( $\mathrm{pH}$ 7.4) with $\mathrm{MgCl}_{2}$ $(3.3 \mathrm{mM})$ and NADPH $(1.3 \mathrm{mM})$ at $37^{\circ} \mathrm{C}$ in a shaking water bath for 1 hour. The incubation was commenced with the addition of NADPH and terminated with the addition of acetonitrile $(5 \mathrm{ml})$. The mixture was spun in a centrifuge at $1700 \mathrm{~g}$ for 5 minutes, the supernatant was transferred to a vacuum centrifuge, and the liquid removed in vacuo. The residue was reconstituted in $0.2 \mathrm{ml}$ water containing methanol $(5 \%)$ and formic acid $(1 \%)$ and analyzed by ultra-high-performance liquid chromatography (UHPLC)-UV-mass spectrometry (MS).

Separation of OH-MVC Isomers by UHPLC-UV-MS. Samples $(10 \mu \mathrm{l})$ were injected onto a Thermo Orbitrap Elite mass spectrometer with an Accela UHPLC-UV system (Thermo Fisher, Waltham, MA). Separation was effected on an Acquity HSS T3 column $(2.1 \times 100 \mathrm{~mm} 1.8 \mu \mathrm{m}$; Waters, Milford, MA) maintained at $45^{\circ} \mathrm{C}$. The mobile phase was composed of $0.1 \%$ formic acid in water (A) and methanol (B) at a flow rate of $0.4 \mathrm{ml} / \mathrm{min}$. The mobile phase gradient was as follows: $0-15$ minute linear gradient from $15 \% \mathrm{~B}$ to $25 \% \mathrm{~B}$, linear gradient to $90 \%$ B at 19 minutes, held at $90 \%$ B for 1 minute, and re-equilibration to initial conditions for 1 minute. The UV detector was monitored from 200 to $400 \mathrm{~nm}$. The mass spectrometer was operated in the positive mode, with source conditions and potentials adjusted to optimize the signal and fragmentation of MVC, and a resolution setting of 30,000.

Biosynthesis of OH-MVC Metabolites. MVC $(20 \mu \mathrm{M})$ was incubated with recombinant CYP3A5 (5.04 nM) in $43 \mathrm{ml} \mathrm{KH}_{2} \mathrm{PO}_{4}(0.1 \mathrm{M})$ containing $\mathrm{MgCl}_{2}$ $(3.3 \mathrm{mM})$ and NADPH $(1.3 \mathrm{mM})$. The incubation was carried out at $37^{\circ} \mathrm{C}$ for 1.75 hours in a shaking water bath. The reaction was terminated with the addition of acetonitrile $(50 \mathrm{ml})$, the mixture was centrifuged at $1700 \mathrm{~g}$ for 5 minutes, and the supernatant was reduced in vacuo to $\sim 10 \mathrm{ml}$. To the remaining material was added formic acid $(0.5 \mathrm{ml})$, acetonitrile $(0.5 \mathrm{ml})$, and water to a total volume of $50 \mathrm{ml}$, and the mixture was spun in a centrifuge for 30 minutes at $40,000 \mathrm{~g}$. The resulting supernatant was applied through a Jasco pump onto a Polaris C18 column $(4.6 \times 250 \mathrm{~mm} ; 5 \mu \mathrm{m})$ at a flow rate of $0.8 \mathrm{ml} / \mathrm{min}$ (Jasco, Easton, MD; Agilent, Santa Clara, CA). After the entire volume was applied, the column was moved to a Thermo-Finnigan Surveyor high-performance liquid chromatography (HPLC)-UV system in line with a linear trap MS system, and a mobile phase gradient was applied to elute the metabolites (Thermo-Finnigan, Waltham, MA). The mobile phase consisted of $0.1 \%$ formic acid in water (A) and acetonitrile (B) at a flow rate of $0.8 \mathrm{ml} / \mathrm{min}$. The composition began at $1 \% \mathrm{~B}$, followed by an immediate increase to $5 \% \mathrm{~B}$ held for 4 minutes, followed by a linear gradient to $40 \%$ B at 100 minutes, an immediate increase to $95 \%$ B to wash the column for 10 minutes, and re-equilibration to initial conditions for 10 minutes. The eluent passed through the UV detector and was then split approximately 10:1 between a fraction collector and the mass spectrometer. Fractions were collected every 20 seconds. Fractions potentially containing metabolites of interest were injected on the UHPLC-MS system described previously to test for identity and apparent purity. Fractions were combined as appropriate, and the solvent was removed in vacuo.

NMR Sample Analysis. Isolated samples and standards were reconstituted in $0.04 \mathrm{ml}$ of methanol- $\mathrm{d}_{4}(100 \%)$ (Cambridge Isotope Laboratories, Andover, MA) and placed in a $1.7 \mathrm{~mm}$ NMR tube under dry argon atmosphere. The ${ }^{1} \mathrm{H}$ and ${ }^{13} \mathrm{C}$ spectra were referenced using residual methanol- $\mathrm{d}_{3}\left({ }^{1} \mathrm{H} \delta=3.35 \mathrm{ppm}\right.$ relative to tetramethylsilane (TMS), $\delta=0.00 ;{ }^{13} \mathrm{C} \delta=49.5 \mathrm{ppm}$ relative to TMS, $\delta=0.00$ ). NMR spectra were recorded on a Bruker Avance $600 \mathrm{MHz}$ (Bruker BioSpin Corporation, Billerica, MA) controlled by Topspin version 3.2 and equipped with a $1.7 \mathrm{~mm}$ TCI CryoProbe. One-dimensional spectra were recorded using an approximate sweep width of $8400 \mathrm{~Hz}$ and a total recycle time of approximately 7 seconds. The resulting time-averaged free induction decays were transformed using an exponential line broadening of $1.0 \mathrm{~Hz}$ to enhance signal to noise. The two-dimensional data were recorded using the standard pulse sequences provided by Bruker BioSpin Corporation. At minimum, a $1000 \times 128$ data matrix was acquired using a minimum of two scans and 16 dummy scans with a spectral width of $10,000 \mathrm{~Hz}$ in the $\mathrm{f} 2$ dimension. The two-dimensional data sets were zero-filled to at least 1000 data points. Postacquisition data processing was performed with either Topspin version 3.2 or MestReNova Mnova 9.1 (Santiago de Campostela, Spain). Quantitation of NMR isolates was performed by external calibration against the ${ }^{1} \mathrm{H}$ NMR spectrum of either a $1 \mathrm{mM}$ maleic acid or $5 \mathrm{mM}$ benzoic acid standard using the ERETIC2 function within Topspin version 3.2.

Metabolism of 2- and 3-OH-MVC Diastereomers to 2,3-DiOH-MVC by Cytochrome P4503A4. OH-MVC isomers $(10 \mu \mathrm{M})$ were each individually incubated with recombinant CYP3A4 $(100 \mathrm{pmol} / \mathrm{ml})$ in $0.5 \mathrm{ml}$ potassium phosphate buffer (100 mM, pH 7.4) containing $\mathrm{MgCl}_{2}$ (3.3 mM) and NADPH $(1.3 \mathrm{mM})$ at $37^{\circ} \mathrm{C}$ for 1 hour. Incubations were terminated by addition of acetonitrile $(2.5 \mathrm{ml})$ and spun in a centrifuge at $1700 \mathrm{~g}$ for 5 minutes. The liquid was removed by vacuum centrifugation in a Genevac and the residue was reconstituted in water containing formic acid $(1 \%)$ and methanol $(5 \%)$. The samples $(5 \mu \mathrm{l})$ were injected onto a Thermo Orbitrap Elite mass spectrometer with an Accela UHPLC-UV system. Separation was effected on an Acquity HSS T3 column $(2.1 \times 100 \mathrm{~mm} 1.8 \mu \mathrm{m})$ and two different mobile phase gradients were used. (Two conditions were used to better ensure that dihydroxy MVC metabolites were indeed the same from incubations of different starting materials.) One mobile phase was $0.1 \%$ formic acid in water (A) and methanol (B), at a temperature of $45^{\circ} \mathrm{C}$. The flow rate was $0.4 \mathrm{ml} / \mathrm{min}$. The mobile phase gradient was as follows: $0-15$ minute linear gradient from $5 \%$ B to $25 \% \mathrm{~B}$, linear gradient to $90 \%$ B at 19 minutes, held at $90 \%$ B for 1 minute, and re-equilibration to initial conditions for 1 minute. The UV detector was monitored from 200 to $400 \mathrm{~nm}$. The second gradient was comprised of mobile phase A of $10 \mathrm{mM}$ ammonium acetate and mobile phase B was methanol. The gradient started at $30 \% \mathrm{~B}$ and increased linearly to $60 \%$ B at 15 minutes, raised to $90 \%$ B at 19 minutes, held for 1 minute, and then re-equilibrated for 1 minute at the initial conditions. The mass spectrometer was operated in the positive mode, and $\mathrm{m} / \mathrm{z} 546$ (corresponding to the protonated molecular ion of diOH-MVC) and its fragment ions were monitored.

Substrate Saturation Incubation in Human Liver Microsomes and Human Recombinant CYP3A4 and CYP3A5. MVC $(1-200 \mu \mathrm{M})$ was incubated in $100 \mathrm{mM}$ potassium phosphate buffer ( $\mathrm{pH} 7.4$ ), $3.3 \mathrm{mM} \mathrm{MgCl}$, and $0.05 \mathrm{mg} / \mathrm{ml}$ microsomal protein (HLM-102, HLM CYP3A5*1/*1, and HLM CYP3A $5 * 3 / * 3$ ) or $10 \mathrm{pmol} / \mathrm{ml}$ recombinant cytochrome $\mathrm{P} 450$ (P450) enzymes (rhCYP3A4 and rhCYP3A5). Incubations were conducted in a $37^{\circ} \mathrm{C}$ heat block for 15 minutes. (These incubation conditions were selected after initial exploration of protein/enzyme concentrations and incubation times that provided linear reaction velocities.) Reactions were terminated by transferring $50 \mu \mathrm{l}$ aliquots of the incubation mixture to $100 \mu \mathrm{l}$ of $50 / 50$ acetonitrile/methanol containing internal standard (mixture of $\mathrm{d}_{5}$-OH-MVC metabolites), followed by vortex mixing. Samples were centrifuged $(1439 g)$ for 10 minutes and $120 \mu \mathrm{l}$ of supernatant was transferred to clean 96 -well plates. The supernatants were dried under $\mathrm{N}_{2}$ and reconstituted in $100 \mu \mathrm{l} 20 / 80$ methanol/0.1\% formic acid in water. Samples were assayed using liquid chromatography-tandem mass spectrometry (LC-MS/MS). Incubations for enzyme kinetic determination were conducted, in triplicate.

Selective Inhibition of CYP3A4 and CYP3A5 Activity in Human Liver Microsomes. MVC $(8 \mu \mathrm{M})$ was incubated with HLM-102, HLM CYP3A5*1/*1, or HLM CYP $3 \mathrm{~A} 5 * 3 / * 3$ in the presence of selective chemical inhibitors ketoconazole ( $1 \mu \mathrm{M}$; for CYP3A) and CYP3cide ( $1 \mu \mathrm{M}$; for CYP3A4), for the determination of CYP3A4 and CYP3A5 isoform contribution. General incubation conditions (protein concentration and incubation time) and sample preparation were as described for the substrate saturation experiments (vide supra).

For incubations with ketoconazole, a mixture containing microsomes, inhibitors, and NADPH or buffer (-NADPH control) was warmed for 5 minutes prior to initiating the reactions by the addition of substrate. Incubations containing CYP3cide were preincubated with microsomes, inactivator, and NADPH or buffer (-NADPH control) for 10 minutes to achieve complete inactivation of CYP3A4 prior to the addition of MVC to initiate the reactions. Chemical inhibition experiments were conducted in triplicate.

LC-MS/MS Method. Supernatants from the terminated incubation mixtures were analyzed using a LC-MS/MS system consisting of an API6500 mass spectrometer ( $\mathrm{AB}$ Sciex, Framingham, MA) equipped with an electrospray source, Agilent 1290 Binary UPLC system (Agilent Technologies, Santa Clara, CA) and a Leap CTC HTS PAL Autosampler (Leap Technologies, Morrisville, 


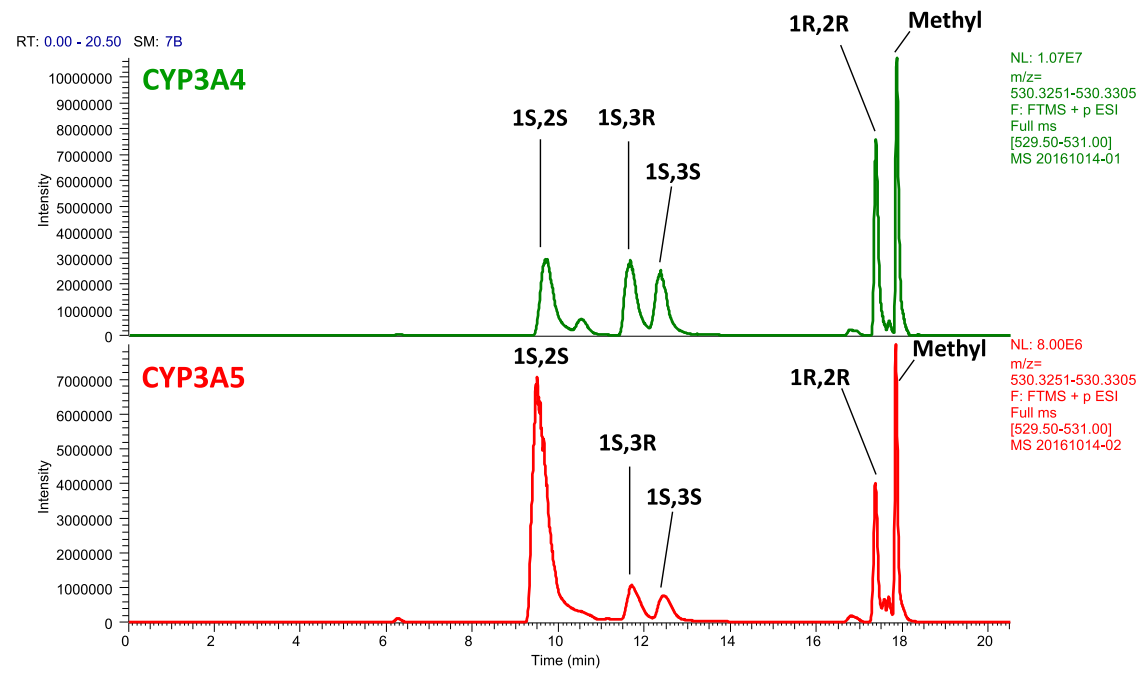

Fig. 1. HPLC-MS chromatograms of metabolites generated in incubations of MVC with cytochrome P450 3A4 and 3A5. The chromatograms are extracted ion traces for $\mathrm{m} / \mathrm{z}$ 530.3278 ( $5 \mathrm{ppm}$ width). The peaks are annotated with the notation for 2- and 3-OH-MVC and hydroxymethyl-MVC. Top panel: CYP3A4. Bottom panel: CYP3A5.

NC). Chromatography was carried out on a Waters Acquity UPLC HSS T3 column $(2.1 \times 100 \mathrm{~mm} ; 1.8 \mu$ particle size $)$ with a mobile phase comprised of water (A) and methanol (B) containing $0.1 \%$ formic acid at a flow rate of $0.4 \mathrm{ml} / \mathrm{min}$. Samples $(0.01 \mathrm{ml})$ were injected and the mobile phase composition was maintained at $20 \% \mathrm{~B}$ for 6.5 minutes, followed by a linear gradient to $35 \% \mathrm{~B}$ over the next 6.5 minutes, and then followed by an increase to $90 \%$ B over 0.5 minutes. It was held at $90 \% \mathrm{~B}$ for another 0.5 minutes before returning to initial conditions and re-equilibrating for 2 minutes. Optimized transitions and parameters of MVC, metabolites, and stable label internal standards used for mass spectrometric detection on the AB Sciex Triple Quad 6500 are listed in Supplemental Table 1. Integration and quantitation of metabolites and internal standard molecule peak areas were performed using Analyst version 1.6.2 (AB Sciex) to derive the analyte to internal standard peak area ratios. Standard curves for the quantitation of metabolite concentration were prepared from plots of area ratio versus concentration and analyzed using a linear regression with either $1 / x$ or $1 / x^{2}$ weighting.
Correlation Analysis with T-5. MVC $(8 \mu \mathrm{M})$ and T-5 $(5 \mu \mathrm{M})$ were incubated in HLMs from individual donors in triplicate. For MVC, general incubation conditions (protein concentration and incubation time) and sample preparation were as described for the substrate saturation experiments. For T-5, experimental and analysis conditions were similar to that described by Li et al. (2014). In general, final incubation mixtures contained $100 \mathrm{mM}$ potassium phosphate buffer ( $\mathrm{pH} 7.4$ ), $3.3 \mathrm{mM} \mathrm{MgCl}$, and $0.1 \mathrm{mg} / \mathrm{ml}$ microsomal protein. Incubations were conducted in a $37^{\circ} \mathrm{C}$ heat block. Reactions were terminated after 20 minutes by transferring $50 \mu \mathrm{l}$ aliquots of the incubation mixture to $100 \mu \mathrm{l}$ of acetonitrile containing internal standard (terfenadine; $2 \mathrm{ng} / \mathrm{ml}$ ), followed by vortex mixing. Samples were centrifuged $(1439 \mathrm{~g})$ for 10 minutes and $120 \mu \mathrm{l}$ of supernatant was transferred to clean 96-well plates. Supernatants from the terminated incubation mixtures were analyzed using the aforementioned LC-MS/MS system. Chromatography was carried out on a Waters Acquity UPLC HSS T3 column $(2.1 \times 50 \mathrm{~mm} ; 1.8 \mu$ particle size $)$ with a mobile phase comprised of water (A) and acetonitrile (B) containing $0.1 \%$ formic acid at a flow
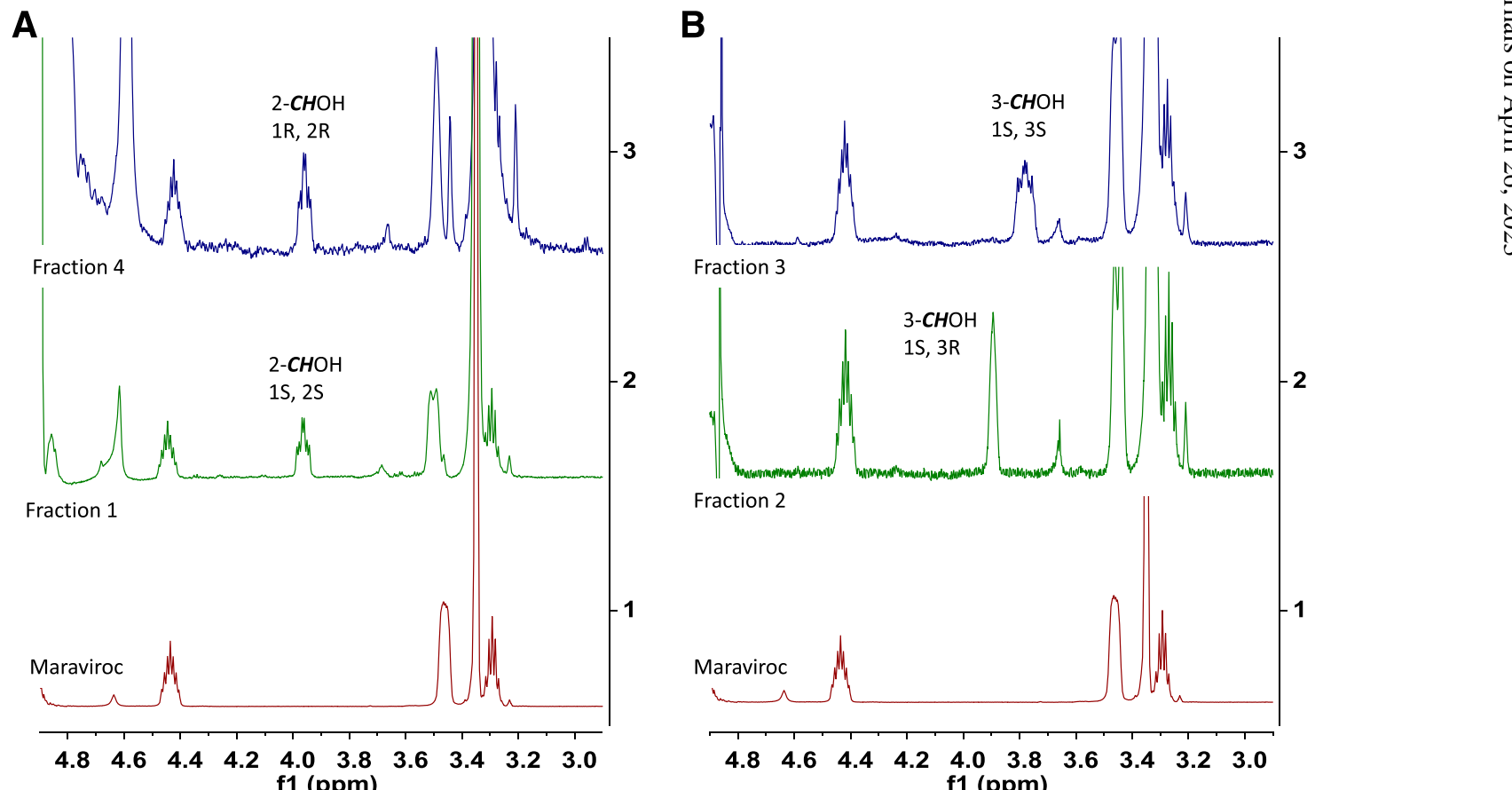

$\begin{array}{llllllllll}4.8 & 4.6 & 4.4 & 4.2 & 4.0 & 3.8 & 3.6 & 3.4 & 3.2 & 3.0\end{array}$ f1 (ppm)

Fig. 2. Abbreviated ${ }^{1} \mathrm{H}$ NMR spectra of (A) MVC (bottom), fraction 1: (1S,2S)-2-OH-MVC (middle), and fraction 4: (1R,2R)-2-OH-MVC (top); (B) MVC (bottom), fraction 2: (1S,3R)-3-OH-MVC (middle), and fraction 3: (1S,3S)-3-OH-MVC (top). 


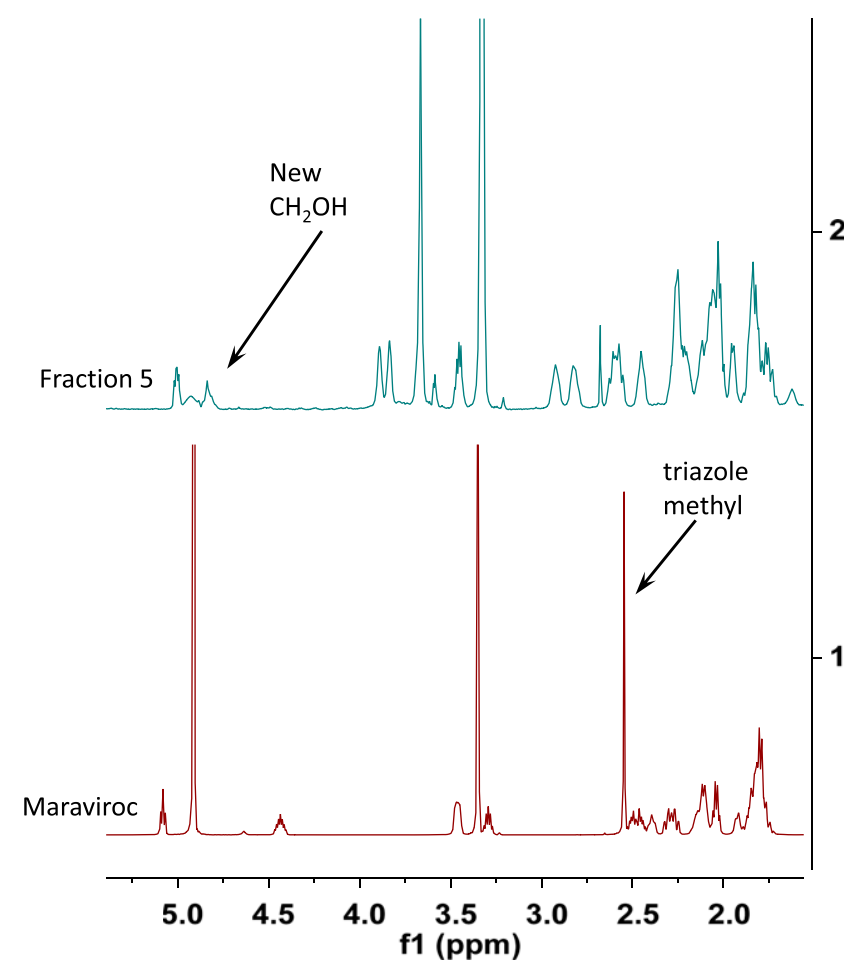

Fig. 3. Abbreviated ${ }^{1}$ H NMR spectrum of hydroxymethyl-MVC and MVC.

rate of $0.4 \mathrm{ml} / \mathrm{min}$. Samples $(0.01 \mathrm{ml})$ were injected and the mobile phase composition was maintained at $5 \% \mathrm{~B}$ for 0.5 minutes followed by a linear gradient to $95 \%$ B over 2 minutes and held at $95 \%$ B for another 0.5 minutes before returning to initial conditions and re-equilibrating to initial conditions for 1 minute. T-5 $\mathrm{N}$-oxide and terfenadine were monitored using mass transitions of $\mathrm{m} / \mathrm{z} 584.2$ 476.2 and $\mathrm{m} / \mathrm{z}$ 472.1-436.1, respectively.

\section{Results}

\section{Profile of OH-MVC Metabolites Generated by Cytochrome P450} 3A4 and 3A5

MVC was incubated with recombinant human P450 3A4 and 3A5 and the incubation extracts were analyzed by HPLC-MS. The extracted ion chromatograms for hydroxylated MVC are shown in Fig. 1. Five metabolites were resolved, observed eluting between 9 and 18 minutes. The first four are 2- and 3-hydroxylations on the difluorocyclohexyl moiety and the fifth one is the hydroxyl on the triazole portion (described subsequently). All five were generated by both enzymes; however, the intensity of the peak eluting at 9.6 minutes was greater in the CYP3A5 incubation than the CYP3A4 incubation. The peaks were shown to coelute with authentic and biosynthesized standards of individual diastereomers of 2- and 3-OH-MVC (Supplemental Figs. 1-5; Supplemental Material). Also, the other four standards of 2- and 3-OH-MVC isomers were shown to elute at different retention times (Supplemental Fig. 6).

\section{Identification of OH-MVC Metabolites}

OH-MVC metabolites were biosynthesized using CYP3A4 and CYP3A5, isolated by HPLC, and regiochemical sites of hydroxylation were assigned using high-resolution MS and NMR spectroscopy. The NMR spectra of metabolites were compared with that of MVC. Exact stereochemical configurations of the hydroxyl isomers on the 4,4difluorocyclohexyl moiety were ultimately assigned by comparison with authentic standards of all eight possible 2- and 3-hydroxy diastereomers.
2-OH-MVC Isomers. The biosynthesis of OH-MVC metabolites using CYP3A5 and CYP3A4 yielded five products. The ones eluting at $\sim 37-40$ minutes (fraction 1) and $\sim 51$ minutes (fraction 4 ) in the preparative purification (Supplemental Fig. 7) were shown to be 2-hydroxy isomers present in CYP3A4 and 3A5 incubation extracts eluting at 9.7 and 17.4 minutes (Fig. 1). The MS $^{\mathrm{n}}$ data showed protonated molecular ions of $\mathrm{m} / \mathrm{z}, 530.3285-530.3294$ (-3 to $-1 \mathrm{ppm})$ and diprotonated molecular ions of $\mathrm{m} / \mathrm{z}$ 265.6677-265.6683 ( -4 to $-1 \mathrm{ppm}$ ), with fragment ions of $\mathrm{m} / \mathrm{z} 405,296,117$, and 106, which are diagnostic of the site of hydroxylation as being on the 4,4-difluorocyclohexyl moiety (Supplemental Figs. 8 and 9). However, distinction between the 2- and 3-positions required the application of NMR spectroscopy.

In fractions 1 and 4 there is a new resonance in the ${ }^{1} \mathrm{H}$ spectrum of each isolate integrating to a single hydrogen at $\delta 3.94(\mathrm{td}, J=10.9$, $4.7 \mathrm{~Hz}$ ) not observed in the ${ }^{1} \mathrm{H}$ spectrum of MVC (Fig. 2A). Multiplicity edited ${ }^{1} \mathrm{H}_{-}{ }^{13} \mathrm{C}$ HSQC data correlate these resonances to a carbon resonance with a chemical shift of $67.6 \mathrm{ppm}$ (Supplemental Figs. 16 and 19). This is consistent with the oxidation of one of the methylenes of the difluorocyclohexane. Initial NMR characterization of isolates from clinical samples assigned these resonances as the $\boldsymbol{H C O H}$ methine of the trans-2-hydroxy MVC isomer. When spectra from fractions 1 and 4 are compared with similarly acquired spectra from a synthetic standard of the 2-hydroxy MVC trans-isomers (fraction $1=1 S, 2 S$ and fraction $4=1 R, 2 R)$ there are no appreciable differences in the ${ }^{1} \mathrm{H} /{ }^{13} \mathrm{C}$ chemical shifts, coupling patterns, or coupling constants of these resonances (Supplemental Figs. 16 and 19). Therefore, the structure of fractions 1 and 4 are assigned as 2-OH-MVC trans-isomers. These structures are further supported by the HPLC coelution of the two biosynthesized

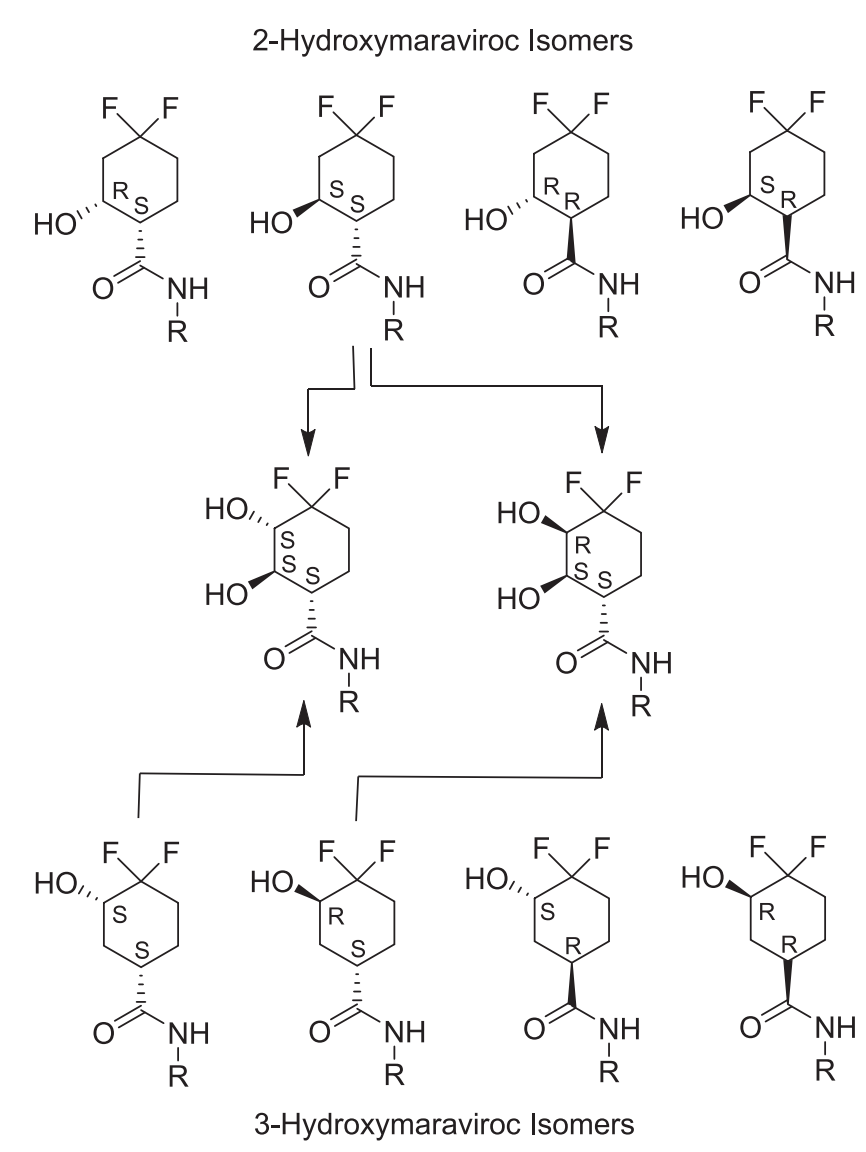

Fig. 4. Metabolism of 2- and 3-OH-MVC diastereomers by cytochrome P450 3A4 to 2,3-DiOH-MVC. 
TABLE 1

Enzyme kinetic parameters for MVC metabolic pathways in pooled HLMs from CYP3A $5 * 1 / * 1$ and CYP3A5*3/*3 donors and recombinant human CYP 3A4 and CYP 3A5

Mean data are from three replicates.

\begin{tabular}{|c|c|c|c|c|c|c|}
\hline Kinetic Parameter ${ }^{a}$ & $(1 S, 2 S)-2-\mathrm{OH}-\mathrm{MVC}$ & $(1 S, 3 R)-3-\mathrm{OH}-\mathrm{MVC}$ & $(1 S, 3 S)-3-\mathrm{OH}-\mathrm{MVC}$ & $(1 R, 2 R)-2-\mathrm{OH}-\mathrm{MVC}$ & Hydroxymethyl-MVC & Total \\
\hline \multicolumn{7}{|l|}{ rhCYP3A4 } \\
\hline$K_{\mathrm{M}} \pm$ S.E. & $10.4 \pm 0.476$ & $10.1 \pm 0.504$ & $10.0 \pm 0.524$ & $10.5 \pm 0.556$ & $10.9 \pm 0.452$ & \\
\hline$V_{\max } \pm$ S.E. & $0.96 \pm 0.0117$ & $1.31 \pm 0.0173$ & $0.81 \pm 0.0112$ & $0.57 \pm 0.00808$ & $3.41 \pm 0.0380$ & \\
\hline $\mathrm{CL}_{\text {int,app }}$ & 0.09 & 0.13 & 0.08 & 0.05 & 0.31 & 0.67 \\
\hline \multicolumn{7}{|l|}{ rhCYP3A5 } \\
\hline$K_{\mathrm{M}} \pm$ S.E. & $8.4 \pm 0.622$ & $7.8 \pm 0.439$ & $7.6 \pm 0.417$ & $8.0 \pm 0.466$ & $8.2 \pm 0.458$ & \\
\hline$V_{\max } \pm$ S.E. & $7.3 \pm 0.139$ & $0.8 \pm 0.0116$ & $0.5 \pm 0.00687$ & $0.8 \pm 0.0115$ & $1.4 \pm 0.0192$ & \\
\hline $\mathrm{CL}_{\text {int,app }}$ & 0.87 & 0.11 & 0.07 & 0.10 & 0.16 & 1.31 \\
\hline \multicolumn{7}{|l|}{ HLM-102 } \\
\hline$K_{\mathrm{M}} \pm$ S.E. & $12.9 \pm 0.477$ & $13.3 \pm 0.534$ & $12.5 \pm 0.584$ & $13.4 \pm 0.517$ & $15.5 \pm 0.707$ & \\
\hline$V_{\max } \pm$ S.E. & $121 \pm 1.26$ & $113 \pm 1.27$ & $89.3 \pm 1.16$ & $75.7 \pm 0.824$ & $216 \pm 2.87$ & \\
\hline $\mathrm{CL}_{\text {int,app }}$ & 9.4 & 8.5 & 7.1 & 5.7 & 14.0 & 44.6 \\
\hline \multicolumn{7}{|l|}{ HLM CYP 3 A $5 * 1 / * 1$} \\
\hline$K_{\mathrm{M}} \pm$ S.E. & $15.6 \pm 0.778$ & $17.4 \pm 1.02$ & $16.9 \pm 0.927$ & $18.0 \pm 1.07$ & $18.6 \pm 1.00$ & \\
\hline$V_{\max } \pm$ S.E. & $245 \pm 3.56$ & $65.6 \pm 1.15$ & $54.3 \pm 0.884$ & $52.5 \pm 0.945$ & $119 \pm 1.95$ & \\
\hline $\mathrm{CL}_{\text {int,app }}$ & 15.6 & 3.8 & 3.2 & 2.9 & 6.4 & 32.0 \\
\hline \multicolumn{7}{|l|}{ HLM CYP 3 A $5 * 3 / * 3$} \\
\hline$K_{\mathrm{M}} \pm$ S.E. & $15.5 \pm 0.779$ & $15.9 \pm 0.589$ & $15.9 \pm 0.714$ & $17.1 \pm 0.807$ & $17.0 \pm 0.696$ & \\
\hline$V_{\max } \pm$ S.E. & $81.3 \pm 1.19$ & $98.7 \pm 1.07$ & $80.2 \pm 1.06$ & $66.8 \pm 0.941$ & $182 \pm 2.22$ & \\
\hline $\mathrm{CL}_{\text {int,app }}$ & 5.2 & 6.2 & 5.0 & 3.9 & 10.7 & 31.1 \\
\hline
\end{tabular}

standards with the authentic $1 S, 2 S$ and $1 R, 2 R$ diastereomers and not the $1 R, 2 S$ or $1 S, 2 R$ diastereomers (Supplemental Figs. 1, 2, and 6). Finally, in a previous report (Abel et al., 2008), these metabolites were ambiguously designated as $\mathrm{H} 7, \mathrm{H} 8, \mathrm{H} 10$, and/or $\mathrm{H} 11$. The present data identifies $(1 S, 2 S)$-2-OHMVC as $\mathrm{H} 8$ and $(1 R, 2 R)$-2-OH-MVC as $\mathrm{H} 11$, which represented $7 \%$ and $5 \%$ of total dose in excreta, respectively (Supplemental Table 2).

3-OH-MVC Isomers. In the aforementioned biosynthesis, two other isolated peaks eluting at $\sim 43-45$ minutes (Supplemental Fig. 7) were shown to be 3-hydroxy isomers present in CYP3A4 and 3A5 incubation extracts eluting at 11.8 and 12.5 minutes (Fig. 1). As with the 2-hydroxyisomers, $\mathrm{MS}^{\mathrm{n}}$ data showed protonated molecular ions of $\mathrm{m} / \mathrm{z}$ 530.3287-530.3291 ( -3 to $-2 \mathrm{ppm})$ and diprotonated molecular ions of $\mathrm{m} / \mathrm{z}, 265.6678-265.6682$ ( -3 to $-2 \mathrm{ppm}$ ), with fragment ions of $\mathrm{m} / \mathrm{z}$ $405,296,117$, and 106, which are diagnostic of the site of hydroxylation as being on the 4,4-difluorocyclohexyl moiety (Supplemental Figs. 10 and 11). NMR spectroscopy was required to delineate the 3-position as the regiochemical site of hydroxylation. As with fractions 1 and 4, there are new resonances in the ${ }^{1} \mathrm{H}$ spectra of fractions 2 and 3 integrating to a single hydrogen at $\delta 3.90$ (broad singlet, fraction 2) and $\delta 3.78$ (ddt, $J=20.1,11.2,4.5 \mathrm{~Hz}$, fraction 3 ) not observed in the ${ }^{1} \mathrm{H}$ spectrum of MVC (Fig. 2B). Multiplicity edited ${ }^{1} \mathrm{H}^{13} \mathrm{C}$ HSQC data correlate these resonances to carbon resonances with chemical shifts of $\delta 67.1$ (fraction 2) and $\delta 69.6$ (fraction 3) (Supplemental Figs. 17 and 18). Again, this is consistent with the oxidation of one of the methylenes of the difluorocyclohexane. When spectra from fractions 2 and 3 are compared with similarly acquired spectra from synthetic standards of the 3-hydroxy MVC isomers there are no appreciable differences in the ${ }^{1} \mathrm{H} /{ }^{13} \mathrm{C}$ chemical shifts, coupling patterns, or coupling constants of these resonances (Fig. 2B). Therefore, the structure of fractions 2 and 3 are assigned as 3-OH-MVC isomers. Unlike the 2-OH-MVC diastereomers, the chemical synthesis of the 3-hydroxy isomers yielded two sets of stereoisomers (cis and trans) but absolute configurations were not known. Absolute stereochemistry of the 3-OH-MVC metabolites was elucidated using a cross metabolism approach (vide infra).

Hydroxymethyl-MVC. The fifth hydroxyl metabolite of MVC generated by CYP3A was biosynthesized and eluted at $\sim 63-65$ minutes (fraction 5) (Supplemental Fig. 7) and corresponded to the metabolite eluting at 17.8 minutes in CYP3A4 and CYP3A5 incubation extracts (Fig. 1; Supplemental Fig. 5). The protonated and diprotonated ions of $\mathrm{m} / \mathrm{z}, 530.3290$ and 265.6680 ( -3 to $-2 \mathrm{ppm}$ ) show addition of a single oxygen. Unlike the other four metabolites previously mentioned, the fragmentation was different (Supplemental Fig. 12). The fragment ion at $\mathrm{m} / \mathrm{z}, 389$ limits the site of hydroxylation to the triazole moiety, since this ion represents no alteration to the rest of the molecule. The ${ }^{1} \mathrm{H}$ spectrum of fraction 5 contains all of the aromatic and aliphatic resonances of MVC except the methyl of the triazole (Fig. 3). Additionally, in the ${ }^{1} \mathrm{H}^{1}{ }^{13} \mathrm{C}$ multiplicity edited HSQC there is a new methylene crosspeak ( $\delta 1 \mathrm{H} \mathrm{4.84/ \delta} 13 \mathrm{C} 54.1$ ) not observed in MVC (Supplemental Fig. 20). These data are consistent with the oxidation of the methyl of the triazole to an alcohol, 4,4-difluoro-N-((S)-3-((1R,3R,5S)-3-(3-(hydroxymethyl)5-isopropyl-4H-1,2,4-triazol-4-yl)-8-azabicyclo[3.2.1] octan-8-yl)-1phenylpropyl)cyclohexane-1-carboxamide. This metabolite corresponds to the previously described H13 metabolite from Abel et al. (2008), which was excreted as $10 \%$ of the dose of MVC (Supplemental Table 2).

\section{Elucidation of the Absolute Stereochemical Configuration of the 3-OH-MVC Metabolites}

At the time of their synthesis, the specific configurations of the four 3-OH-MVC diastereomers was not known; however, the cis- and trans-configurations were known. One diastereomer in each of these preparations matched a CYP3A-generated metabolite. Since the absolute configuration of all four 2-OH-MVC isomers was already known, an approach was taken wherein all eight synthetic 2- and 3-OH-MVC isomers were subjected to further metabolism by CYP3A4 with the intent that specific 2,3-diOH-MVC metabolites would be generated from the individual 2- and 3-OH-MVC substrates and would have identical HPLC-MS properties. If this were the case, then using the known configuration at the 1-position of the 2-OH-MVC, which would be unchanged in a 2,3-dihydroxy metabolite, the configuration of the 1-position of the 3-hydroxy metabolite that yields the same 2,3-dihydroxy metabolite can be inferred. Since this will be one of a pair of diastereomers known to be either cis or trans, the 3-position absolute configuration can be concluded (see Fig. 4). 
(1S,2S)-2-hydroxymaraviroc

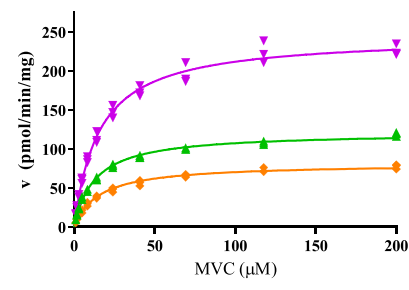

(1S,3R)-3-hydroxymaraviroc

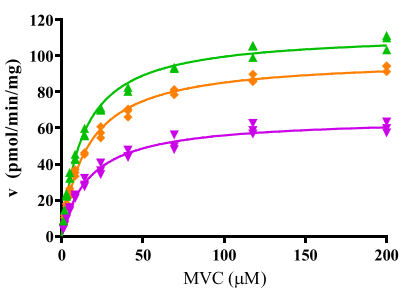

(1S,3S)-3-hydroxymaraviroc

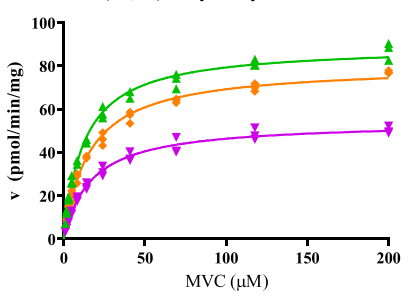

(1R,2R)-2-hydroxymaraviroc

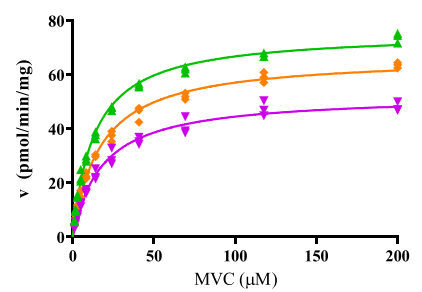

Hydroxymethylmaraviroc

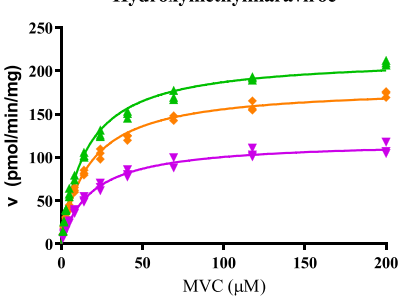

(1S,2S)-2-hydroxymaraviroc

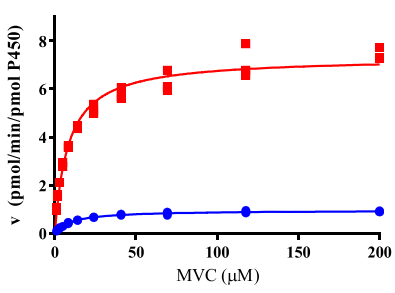

(1S,3R)-3-hydroxymaraviroc

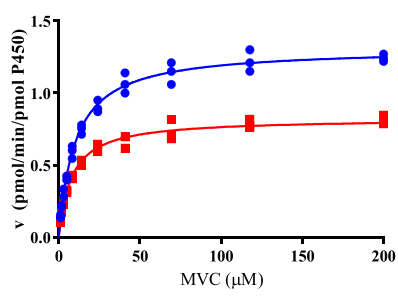

(1S,3S)-3-hydroxymaraviroc

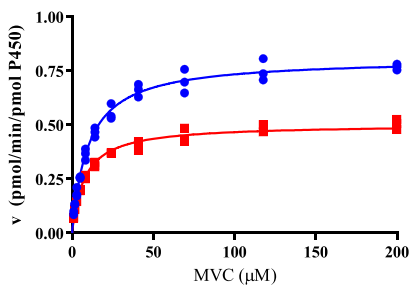

(1R,2R)-2-hydroxymaraviroc

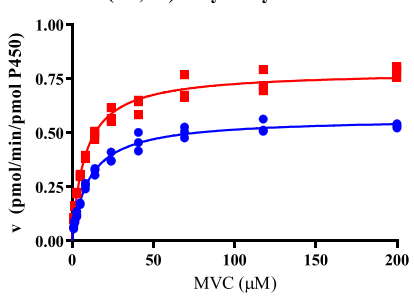

t- HLM102

* HLM 3 A $5 * 1 /{ }^{*}$

$\rightarrow$ HLM 3 A $5 * 3 / * 3$
Hydroxymethylmaraviroc

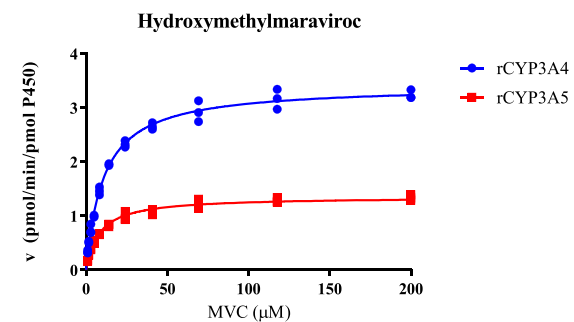

Fig. 5. Substrate saturation curves of $(1 S, 2 S),(1 R, 2 R)$, $(1 S, 3 S),(1 S, 3 R)$, and hydroxymethyl-MVC formation in pooled HLMs, pooled CYP3A $5 * 1 / * 1$ HLMs, pooled CYP3A5*3/*3 liver microsomes, and recombinant human CYP3A4 and CYP3A5. Plots on the left are in HLMs and plots on the right are in recombinant systems.
The cis-3-hydroxy isomer eluting at 12.4 minutes (Fig. 1) and (1S,2S)2-OH-MVC yielded the same diOH-MVC metabolite eluting at 8.9 minutes (Supplemental Fig. 13) with a protonated molecular ion at $\mathrm{m} / \mathrm{z}, 546.3259(0.9 \mathrm{ppm})$. The $\mathrm{MS}^{\mathrm{n}}$ spectra showed fragment ions of $\mathrm{m} / \mathrm{z}$ $421,312,117$, and 106, which narrows the site of hydroxylation to the 4,4-difluorocyclohexyl moiety (Supplemental Fig. 14). To confirm that this dihydroxy metabolite generated by the 2- and 3-OH-MVC substrates is the same, the sample was analyzed by HPLC using a different mobile phase condition, and the peaks continued to coelute (Supplemental Fig. 13). The known configuration of the 2-OH-MVC substrate at the 1-position is $S$. The cis-3-OH-MVC substrate that yields the same dihydroxy metabolite were either $1 S, 3 S$ or $1 R, 3 R$, but since it yielded the same dihydroxy metabolite as the $(1 S, 2 S)-2-\mathrm{OH}-\mathrm{MVC}$ isomer, it can be concluded that the CYP3A-generated 3-OH-MVC metabolite that elutes at 12.4 minutes is the $1 S, 3 S$ isomer (Fig. 8). An identical logic and approach was applied to elucidate the absolute stereochemistry of the 3-OH-MVC metabolite eluting at 11.8 minutes (Fig. 1). In this case, this 3-hydroxy metabolite was shown to be the $1 S, 3 R$ isomer (Fig. 8). The $1 S, 3 S$ and $1 S, 3 R$ metabolites correspond to those designated as $\mathrm{H} 7$ and $\mathrm{H} 10$ in the human radiolabel mass balance study, which represented $8 \%$ and $9 \%$ of dose (Abel et al., 2008) (Supplemental Table 2) 
(1S,2S)-2-hydroxymaraviroc

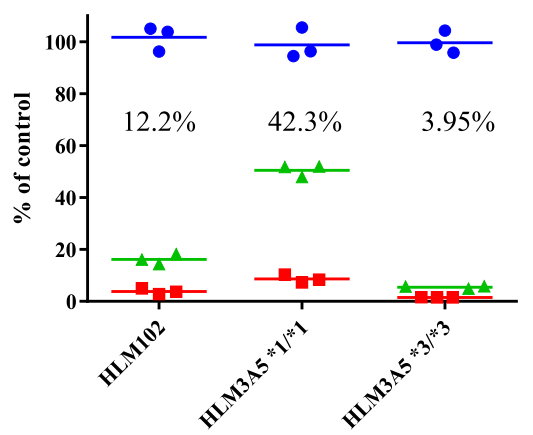

(1R,2R)-2-hydroxymaraviroc

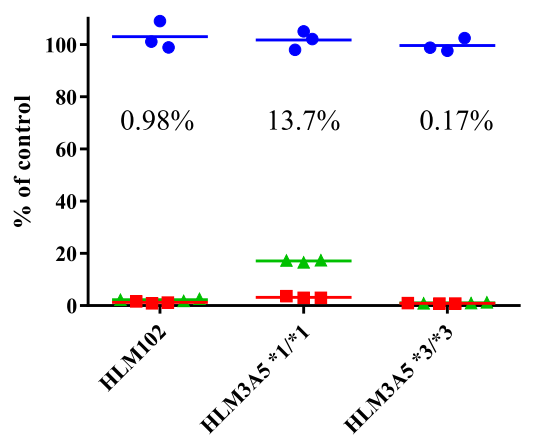

(1S,3R)-3-hydroxymaraviroc

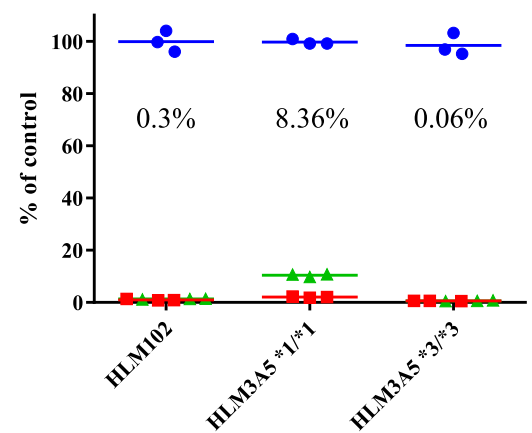

Hydroxymethylmaraviroc

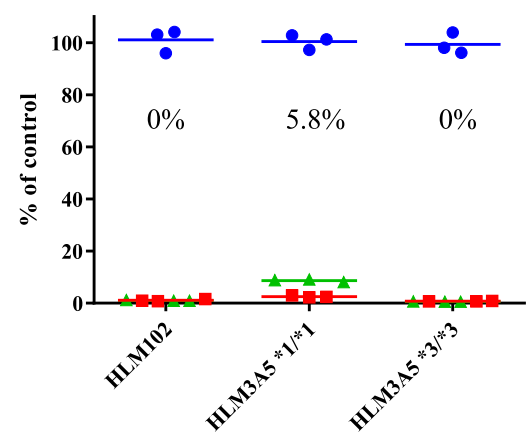

(1S,3S)-3-hydroxymaraviroc

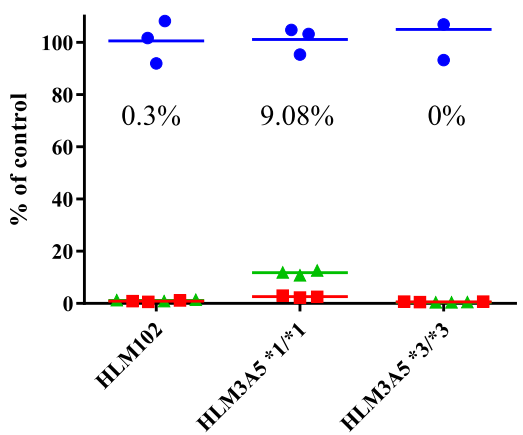

$\triangle$ CYP3cide

- Ketoconazole

- Control

Fig. 6. Formation of $(1 S, 2 S)-2-\mathrm{OH}-\mathrm{MVC},(1 R, 2 R)-2-\mathrm{OH}-\mathrm{MVC},(1 S, 3 S)-3-\mathrm{OH}-\mathrm{MVC},(1 S, 3 R)-3-\mathrm{OH}-\mathrm{MVC}$, and hydroxymethyl-MVC in pooled HLMs, pooled CYP3A5*1/*1 HLMs, and pooled CYP3A5*3/*3 liver microsomes in the presence and absence of selective CYP3A and CYP3A4 inhibitors, ketoconazole and CYP3cide, respectively. Percent values represent the calculated contribution by CYP3A5.

\section{Enzyme Kinetics of Maraviroc in Human Liver Microsomes and} Recombinant Human Cytochrome P450s

Enzyme kinetic parameters and intrinsic clearance values, determined from the formation of the five metabolites of MVC in $\mathrm{rCYP} 3 \mathrm{~A} 4$, rCYP3A5, HLM-102, HLM CYP3A $5 * 1 / * 1$, and HLM CYP3A5*3/*3, are listed in Table 1 and substrate saturation data are presented graphically in Fig. 5. The results for all metabolites fit to the Michaelis-Menten model. In general, the estimated $K_{\mathrm{M}}$ value for each metabolite was comparable to each other within each enzyme system and generally ranged from 7 to $19 \mu \mathrm{M}$. However, the apparent intrinsic clearance $\left(\mathrm{CL}_{\text {int,app }}\right)$ provided an initial indication of which recombinant $\mathrm{P} 450$ and genotype is preferential to the formation of each MVC metabolite. While all the metabolites are formed by CYP3A4 and CYP3A5 to a certain extent, $(1 S, 2 S)-2-\mathrm{OH}-\mathrm{MVC}$ is predominately formed by rCYP3A5 and HLM CYP3A5*1/*1 with values for $\mathrm{CL}_{\text {int,app }}$ of $0.87 \mu \mathrm{l} / \mathrm{min}$ per picomole $\mathrm{P} 450$ and $15.6 \mu \mathrm{l} / \mathrm{min}$ per milligram, respectively. Hydroxymethyl-MVC is mainly formed by rCYP3A4, HLM-102, and CYP3A5*3/*3 with $\mathrm{CL}_{\text {int,app }}$ of $0.31 \mu \mathrm{l} / \mathrm{min}$ per picomole $\mathrm{P} 450,14.0 \mu \mathrm{l} / \mathrm{min}$ per milligram, and $10.7 \mu \mathrm{l} / \mathrm{min}$ per milligram, respectively.

\section{Selective Inhibition of CYP3A4 and CYP3A5}

MVC was incubated in the presence and absence of ketoconazole $(2 \mu \mathrm{M})$ and CYP3cide $(1 \mu \mathrm{M})$ in HLMs from either a 50-donor pool or pooled CYP3A5*1/*1 and CYP3A5*3/*3 donors. The impact on the rate of formation of each metabolite was examined to estimate the relative contributions of CYP3A4 and CYP3A5. Inhibition data are listed in Supplemental Table 3.

For the HLM-102 and CYP3A $5 * 3 / * 3$ pools (CYP3A5 poor metabolizer), the estimated CYP3A5 contributions were similar across the five metabolites with less than $12 \%$ by CYP3A5. In the CYP3A $5 * 1 / * 1$ pool (CYP3A5 EM), the greatest CYP3A5 contribution was observed only for $(1 S, 2 S)-2-\mathrm{OH}-\mathrm{MVC}(42 \%)$. The contribution by CYP3A5 was relatively low $(<13 \%)$ for the other four metabolites (see Fig. 6).

\section{Correlation Analysis}

T-5 oxidation to an $N$-oxide metabolite by CYP3A5 was first reported by $\mathrm{Li}$ et al. (2014) as a reaction that is highly correlated with the CYP3A5 genotype and CYP3A5 expression levels in HLMs and hepatocytes. Formation of the five OH-MVC metabolites were compared with T-5 $\mathrm{N}$-oxide formation in individual HLM donors genotyped to be CYP3A5*1/*1, CYP3A5*1/*3, and CYP3A5*3/*3 expressers. A positive correlation with T-5 $N$-oxide was observed for the $(1 S, 2 S)-2$ hydroxy metabolite (the Pearson $r$ value of the correlation was $0.81, P<$ 0.0001) (Fig. 7), while the other four metabolites had no correlation (Supplemental Fig. 15). This suggests that the CYP3A5 genotype has involvement in the metabolic pathway of $(1 S, 2 S)-2-\mathrm{OH}-\mathrm{MVC}$ and not the other metabolites.

\section{Summary of CYP3A Enzyme Contribution to Maraviroc Metabolism Assignment}

Incorporating the estimated $\mathrm{CL}_{\text {int,app }}$ and fraction of CYP3A5 inhibition of each OH-MVC metabolite in HLMs, the total fraction of metabolism contributed by CYP3A5 can be estimated in various batches of HLMs. The in vitro $\mathrm{CL}_{\text {int,app }}$ values in CYP3A5*1/*1 HLM were calculated as the ratio of $V_{\max }$ to $K_{\mathrm{M}}$, where $V_{\max }$ and $K_{\mathrm{M}}$ are kinetic parameters determined in Table 1 , and $\mathrm{CL}_{\text {int,app }}$ is the intrinsic clearance for metabolite, and $\mathrm{CL}_{\text {int,app,all }}$ is the sum of in vitro intrinsic clearance 


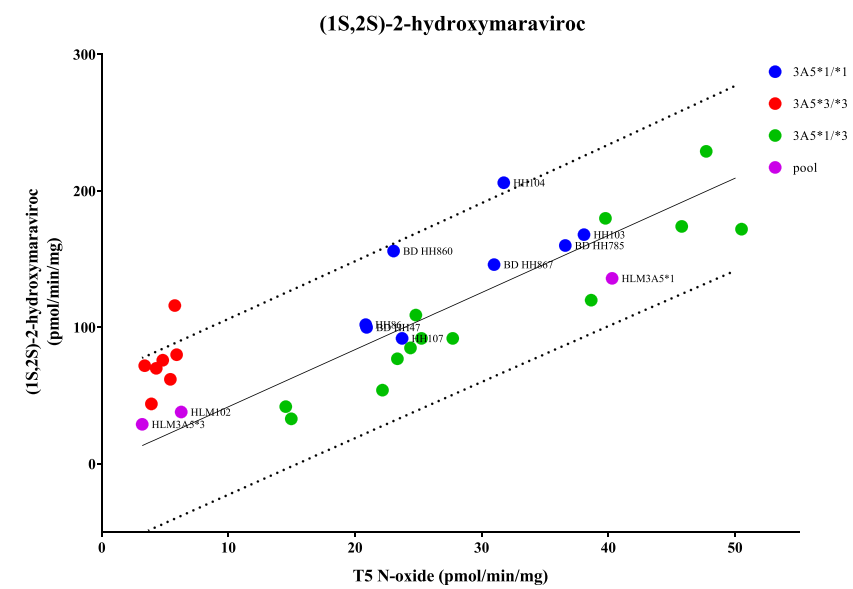

Fig. 7. Activity correlation of $(1 S, 2 S)-2-\mathrm{OH}-\mathrm{MVC}$ formation to T-5 $\mathrm{N}$-oxide formation in pooled and individual CYP $3 \mathrm{~A} 5 * 1 / * 1, \mathrm{CYP} 3 \mathrm{~A} 5 * 1 / * 3$, and CYP3A5*3/*3 liver microsomes. HLM102 represents a 50-donor HLM pool. The dotted lines of regression represent the $90 \%$ prediction bands. The Pearson $r$ correlation value was $0.810(P<0.0001)$

values for all metabolites. The fraction of clearance $\left(f_{C L}\right)$ by each metabolite is calculated as the $\mathrm{CL}_{\text {int,app }}$ of each metabolite divided by the total $\mathrm{CL}_{\text {int,app }}$ for MVC. The fraction of CYP3A5 inhibition was determined from the chemical inhibition study in HLMs incorporating ketoconazole and CYP3cide (Supplemental Table 3). The fraction metabolized $\left(f_{m}\right)$ by CY3A5 for each metabolite is calculated as $f_{C L}$ multiplied by the fraction of CYP3A5 inhibition. The total fraction metabolized by CYP3A5 in the *1/*1 genotype is estimated to be 0.25 (Table 2).

Based on the combined results from enzyme kinetics, HLM chemical inhibition, and correlation analysis assays, CYP3A5 was consistently predicted by both HLMs and recombinant human $\mathrm{P} 450$ s to contribute the formation of $(1 S, 2 S)$-2-OH-MVC while the other four hydroxymethyl-MVC metabolites were predominantly generated by CYP3A4.

\section{Discussion}

The application of metabolite biosynthesis using P450 enzymes, followed by NMR spectroscopy to identify regiochemical sites of metabolism and to quantitate the concentration of the material such that it can be used as an analytical standard, is a powerful and facile approach to conducting drug metabolism studies. In this work, we began by biosynthesizing and isolating the five major CYP3A-generated hydroxyl metabolites of MVC. These had previously been identified, but only reported as Markush structures (Abel et al., 2008). These biosynthesized metabolites were then used as analytical standards to carry out work to quantitatively assess the potential role of CYP3A5 in the metabolism of MVC. They were also used to justify investment in a lengthy and expensive chemical synthesis of all eight possible isomers of 2- and 3-OH-MVC such that the absolute configuration of these metabolites could be ascertained.

Previous in vitro work reported by $\mathrm{Lu}$ et al. (2012) proposed that CYP3A5 has a major role in the metabolism of MVC. However, quantitation of the metabolites done in that work as well as analysis of human plasma samples (Lu et al., 2014) was based on mass spectral response using the 4-hydroxyphenyl analog of MVC that was not the actual metabolite. Because 4-hydroxyphenyl MVC is structurally dissimilar to the identified $(1 S, 2 S)$-2-OH-MVC metabolite in terms of the electronic character of the phenolic versus the aliphatic hydroxyl moieties, it is likely that the estimation of the M1 concentration reported by $\mathrm{Lu}$ et al. (2014) would show some bias compared with the actual concentrations present in clinical plasma samples. The claim of a prominent role for CYP3A5 by Lu et.al. (2012) was based primarily on work in recombinant enzymes without the benefit of a relative activity factor or intersystem extrapolation factor for this enzyme that is necessary to quantitatively relate rate data from recombinant heterologously expressed enzymes to activities in liver microsomes or in vivo (Proctor et al., 2004; Chen et al., 2011). The lack of a suitable activity factor makes translating the in vitro results to in vivo contribution problematic.

In the present work, we have used the actual metabolite standards in calibration curves required for quantitation by HPLC-MS. We have also employed the CYP3A4 selective inactivator cyp3cide to quantitatively delineate the role of CYP3A4 versus CYP3A5 in pooled liver microsomes from CYP3A $5 * 1 / * 1$ donors to get a more reliable estimate of the contribution of CYP3A5 to all five of the major in vitro metabolites. From these data, it can be clearly concluded that CYP3A5 has a contributing role in the generation of the $(1 S, 2 S)-2-\mathrm{OH}-\mathrm{MVC}$ metabolite-which is most likely the metabolite 1 described by Lu et al. (2012) - but not the other metabolites. By defining the individual metabolic pathways, determining the intrinsic clearance of their formation to gain knowledge of the fraction that each contributes to the whole, and then delineating the role of CYP3A4 versus CYP3A5 to each of the metabolites, we have reconstructed the total picture of MVC metabolism to show that CYP3A5 contributes approximately $25 \%$ to the metabolism of MVC in CYP3A5 EMs (Fig. 8). This is similar to a previous estimate (32\%) made by monitoring depletion of MVC in HLMs from a CYP3A5*1/*1 donor (Tseng et.al., 2014).

CYP3A5 is subject to a genetic polymorphism wherein a larger percentage of individuals of African descent express the enzyme (Roy et al., 2005; Lamba et al., 2012). Thus, drugs that are shown to be substrates of CYP3A5 have the possibility to show lower exposure

TABLE 2

Estimation of CYP3A5 fraction metabolized in HLMs pooled from CYP3A5 extensive metabolizers

\begin{tabular}{|c|c|c|c|c|}
\hline & HLM CYP $3 A 5^{*} 1 / * 1 \mathrm{Cl}_{\text {int,app }}$ & $\mathrm{f}_{\mathrm{CL}}$ & $\begin{array}{l}\text { Fraction of CYP3A5 Contribution Estimated by Differential Inhibition by } \\
\text { CYP3cide and Ketoconazole }\end{array}$ & Estimated $\mathrm{f}_{\mathrm{m}}$ for CYP3A5 \\
\hline \multicolumn{5}{|c|}{$\mu \mathrm{l} / \mathrm{min} / \mathrm{mg}$} \\
\hline$(1 S, 2 S)-2-\mathrm{OH}-\mathrm{MVC}$ & 15.6 & 0.5 & 0.42 & 0.21 \\
\hline$(1 S, 3 R)-3-\mathrm{OH}-\mathrm{MVC}$ & 3.8 & 0.1 & 0.08 & 0.01 \\
\hline$(1 S, 3 S)-3-\mathrm{OH}-\mathrm{MVC}$ & 3.2 & 0.1 & 0.09 & 0.01 \\
\hline$(1 R, 2 R)-2-\mathrm{OH}-\mathrm{MVC}$ & 2.9 & 0.1 & 0.14 & 0.01 \\
\hline Hydroxymethyl-MVC & 6.4 & 0.2 & 0.06 & 0.01 \\
\hline $\mathrm{CL}_{\text {int,app,all }}$ & 31.9 & & & \\
\hline Total & & & & 0.25 \\
\hline
\end{tabular}

$\mathrm{f}_{\mathrm{CL}}$, fraction of total metabolic clearance; $\mathrm{f}_{\mathrm{m}}$, fraction metabolized. 


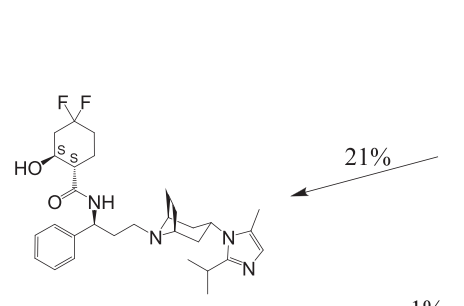

(1S,2S)-2-hydroxymaraviroc

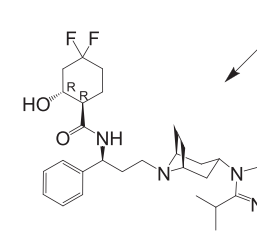

$(1 \mathrm{R}, 2 \mathrm{R})-2$-hydroxymaraviroc
YF

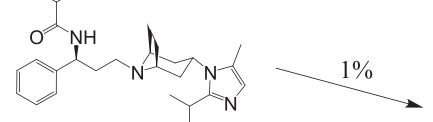

maraviroc

$\%$
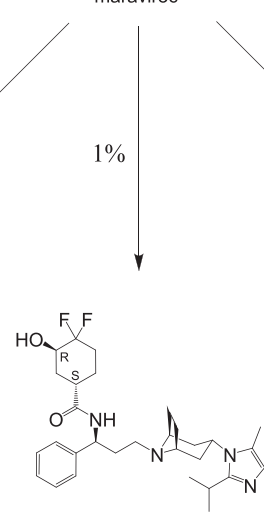

$(1 \mathrm{~S}, 3 \mathrm{R})$-3-hydroxymaraviroc

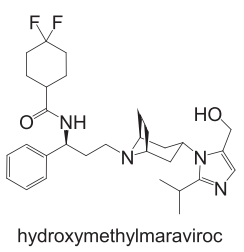

1

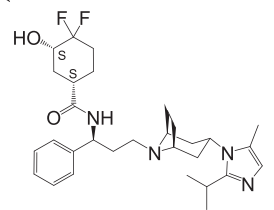

(1S,3S)-3-hydroxymaraviroc
Fig. 8. Metabolism scheme of MVC by CYP3A4 and CYP3A5. The percentages denote the estimated CYP3A5 contribution to each metabolite in CYP3A5*1/*1 HLMs. in individuals possessing one or two copies of the CYP3A $5 * 1$ allele (i.e., IMs and EMs). However, the quantitative impact of CYP3A5 must be weighed against the role that other enzymes, especially CYP3A4, have in the overall metabolism, as well as nonmetabolic clearance pathways such as the considerable portion of MVC excreted unchanged in urine (Abel et al., 2008). To date, all drugs that have been shown to be metabolized by CYP3A5 are also metabolized by CYP3A4; therefore, quantitatively assessing the role of CYP3A5 in the metabolism of a compound has been challenging up until the introduction of the CYP3A4 selective inactivator cyp3cide (Walsky et al., 2012), which permits the activities of CYP3A4 and CYP3A5 to be separated in intact in vitro models of drug metabolism (i.e., HLMs or hepatocytes). The results from this work suggest that CYP3A5 would play a minor but measurable role in the metabolic clearance of MVC in CYP3A5 EM individuals. This has been shown in the clinic wherein CYP3A5 EM subjects have been shown to have between $26 \%$ and $41 \%$ lower exposure to MVC and a higher $(1 S, 2 S)-2-\mathrm{OH}-\mathrm{MVC}$ to MVC exposure ratio, consistent with estimates made from the in vitro data (M. Vourvahis, manuscript in preparation; Lu et al., 2014). However, it is also the observation that these lower exposures are still in excess of exposures associated with near-maximal MVC virologic efficacy, which may explain why there was no observation of decreased efficacy in CYP3A5 EM patients in the phase 3 efficacy trials of MVC (McFadyen et al., 2008; Vourvahis et al., 2015). Additionally, it should be noted that in clinical practice, many HIV patients receive MVC in the presence of CYP3A-inhibiting protease inhibitors. Such drugs are potent inhibitors of both CYP3A4 and CYP3A5 (Ernest et.al. 2005; Granfors et.al. 2006), and therefore this could potentially diminish the overall role of CYP3A (both 3A4 and 3A5) in the overall metabolism of MVC in patients receiving these drugs.

Overall, these results demonstrate that CYP3A5 can contribute partially to the generation of the $(1 S, 2 S)$-2-OH-MVC metabolite. The use of biosynthesized metabolites with quantitative NMR spectroscopy served well to enable the subsequent $\mathrm{P} 450$ reaction phenotyping experiments needed to quantitatively evaluate the relative roles of CYP3A4 and 3A5 in the metabolism of MVC. Such an approach can be employed to tackle quantitative in vitro drug metabolism experiments for other compounds, even when synthetic standards of metabolites are difficult or impossible to obtain.

\section{Acknowledgments}

We thank the following individuals: Olivier Dirat of the Pharmaceutical Research and Development Department at Pfizer, Sandwich, United Kingdom, for coordination of the synthesis of 2- and 3-OH-MVC standards conducted at Peakdale Molecular; Jan Szeliga of the Pharmaceutical Research and Development Department, Pfizer, Groton, CT, for preparative chromatography of the four synthetic 3-OH-MVC isomers; and Mark Savage, Torren Peakman, and Angus Nedderman, formerly of Pfizer, Sandwich, United Kingdom, who conducted excellent early work on MVC metabolism that was used as a starting point for these investigations.

\section{Authorship Contributions}

Participated in research design: Tseng, Fate, Walker, Goosen, Obach. Conducted experiments: Tseng, Walker, Obach.

Performed data analysis: Tseng, Fate, Walker, Obach.

Wrote or contributed to the writing of the manuscript: Tseng, Fate, Walker, Goosen, Obach.

\section{References}

Abel S, Back DJ, and Vourvahis M (2009) Maraviroc: pharmacokinetics and drug interactions. Antivir Ther 14:607-618.

Abel S, Russell D, Whitlock LA, Ridgway CE, Nedderman ANR, and Walker DK (2008) Assessment of the absorption, metabolism and absolute bioavailability of maraviroc in healthy male subjects. Br J Clin Pharmacol 65 (Suppl 1):60-67.

Chen Y, Liu L, Nguyen K, and Fretland AJ (2011) Utility of intersystem extrapolation factors in early reaction phenotyping and the quantitative extrapolation of human liver microsomal intrinsic clearance using recombinant cytochromes P450. Drug Metab Dispos 39:373-382.

Dorr P, Westby M, Dobbs S, Griffin P, Irvine B, Macartney M, Mori J, Rickett G, Smith-Burchnell C, Napier C, et al. (2005) Maraviroc (UK-427,857), a potent, orally bioavailable, and selective small-molecule inhibitor of chemokine receptor CCR5 with broad-spectrum anti-human immunodeficiency virus type 1 activity. Antimicrob Agents Chemother 49:4721-4732.

Ernest CS, II, Hall SD, and Jones DR (2005) Mechanism-based inactivation of CYP3A by HIV protease inhibitors. J Pharmacol Exp Ther 312:583-591.

Granfors MT, Wang JS, Kajosaari LI, Laitila J, Neuvonen PJ, and Backman JT (2006) Differ ential inhibition of cytochrome P450 3A4, 3A5 and 3A7 by five human immunodeficiency virus (HIV) protease inhibitors in vitro. Basic Clin Pharmacol Toxicol 98:79-85.

Hyland R, Dickins M, Collins C, Jones H, and Jones B (2008) Maraviroc: in vitro assessment of drug-drug interaction potential. Br J Clin Pharmacol 66:498-507.

Lamba J, Hebert JM, Schuetz EG, Klein TE, and Altman RB (2012) PharmGKB summary: very important pharmacogene information for CYP3A5. Pharmacogenet Genomics 22: $555-558$.

Li X, Jeso V, Heyward S, Walker GS, Sharma R, Micalizio GC, and Cameron MD (2014) Characterization of T-5 $\mathrm{N}$-oxide formation as the first highly selective measure of CYP3A5 activity. Drug Metab Dispos 42:334-342.

Lu Y, Fuchs EJ, Hendrix CW, and Bumpus NN (2014) CYP3A5 genotype impacts maraviroc concentrations in healthy volunteers. Drug Metab Dispos 42:1796-1802.

Lu Y, Hendrix CW, and Bumpus NN (2012) Cytochrome P450 3A5 plays a prominent role in the oxidative metabolism of the anti-human immunodeficiency virus drug maraviroc. Drug Metab Dispos 40:2221-2230. 
McFadyen L, Jacqmin P, Wade JR, and Weatherley B (2008) Maraviroc exposure-efficacy $(<50$ copies $/ \mathrm{mL}$ ) analysis in HIV-1-infected treatment-naive subjects-ITT population (MERIT study), in Proceedings of the 17th International AIDS Conference, Mexico City, Mexico, August 3-8. Poster TUPE0053.

Michael NL, Chang G, Louie LG, Mascola JR, Dondero D, Birx DL, and Sheppard HW (1997) The role of viral phenotype and CCR-5 gene defects in HIV-1 transmission and disease progression. Nat Med 3:338-340.

Philpott SM (2003) HIV-1 coreceptor usage, transmission, and disease progression. Curr HIV Res 2:217-227.

Proctor NJ, Tucker GT, and Rostami-Hodjegan A (2004) Predicting drug clearance from recombinantly expressed CYPs: intersystem extrapolation factors. Xenobiotica 34:151-178.

Roy JN, Lajoie J, Zijenah LS, Barama A, Poirier C, Ward BJ, and Roger M (2005) CYP3A5 genetic polymorphisms in different ethnic populations. Drug Metab Dispos 33:884-887.

Tseng E, Walsky RL, Luzietti RA, Jr, Harris JJ, Kosa RE, Goosen TC, Zientek MA, and Obach RS (2014) Relative contributions of cytochrome CYP3A4 versus CYP3A5 for CYP3A-cleare drugs assessed in vitro using a CYP3A4-selective inactivator (CYP3cide). Drug Metab Dispos 42:1163-1173.
Vourvahis M, Fang J, Checchio T, Milton A, Weatherley B, McFadyen L, and Heera J (2013) Pharmacokinetics, safety, and tolerability of maraviroc in HIV-negative subjects with impaired renal function. HIV Clin Trials 14:99-109.

Vourvahis M, McFadyen L, Heera J, and Clark A (2015) Clinical relevance of CYP3A5 genotype on maraviroc exposures. Drug Metab Dispos 43:771-772.

Walsky RL, Obach RS, Hyland R, Kang P, Zhou S, West M, Geoghegan KF, Helal CJ, Walker GS, Goosen TC, et al. (2012) Selective mechanism-based inactivation of CYP3A4 by CYP3cide (PF-04981517) and its utility as an in vitro tool for delineating the relative roles of CYP3A4 versus CYP3A5 in the metabolism of drugs. Drug Metab Dispos 40: $1686-1697$.

Address correspondence to: Gwendolyn D. Fate, Athena DMPK LLC, 29 Willow Lane, East Lyme, CT, 06333. E-mail: gwenfate@gmail.com; or R. Scott Obach, Pfizer Inc., Eastern Point Rd, Groton, CT 06340. E-mail: r.scott.obach@pfizer.com 